\title{
Sanggenon C protects against cardiomyocyte hypoxia injury by increasing autophagy
}

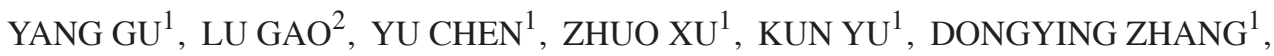 \\ ${\text { GANG } \text { ZHANG }^{1} \text { and XIWEN ZHANG }}^{1}$ \\ ${ }^{1}$ Department of Cardiology, Huai'an First People's Hospital, Nanjing Medical University, Huai'an, Jiangsu 223300;
${ }^{2}$ Department of Cardiology, The First Affiliated Hospital of Zhengzhou University, Zhengzhou, Henan 450052, P.R. China
}

Received January 9, 2017; Accepted August 4, 2017

DOI: $10.3892 / \mathrm{mmr} .2017 .7646$

\begin{abstract}
Sanggenon $\mathrm{C}$ is isolated from Morus alba, a plant that has been used for anti-inflammatory purposes in Oriental medicine. Little is known about the effect of Sanggenon C on cardiomyocyte hypoxia injury. This study, using $\mathrm{H} 9 \mathrm{c} 2$ rat cardiomyoblasts, was designed to determine the effects of Sanggenon $\mathrm{C}$ on cardiomyocyte hypoxia injury. Inflammatory cytokine levels were measured by reverse transcription-polymerase chain reaction, reactive oxygen species were measured by 2',7'-dichlorofluorescin diacetate fluorescent probe, autophagy was detected using the LC3II/I ratio and cell apoptosis was detected by TUNEL staining. The molecular mechanisms underlying Sanggenon C-induced cyto-protection were also determined by western blotting, especially the possible involvement of autophagy and AMP-activated protein kinase (AMPK). Results indicated that samples pretreated with different concentrations of Sanggenon $\mathrm{C}(1,10$ and $100 \mu \mathrm{M})$ reduced the expression levels of pro-inflammatory cytokines, including tumor necrosis factor $\alpha$, interleukin (IL)-1 and IL-6, under hypoxia. The beneficial effects of Sanggenon $C$ were also associated with reduced levels of reactive oxygen species generation and increased levels of antioxidant nitric oxide and superoxide dismutase. Sanggenon C enhanced hypoxia-induced autophagy as evidenced by the increased expression levels of autophagy-associated proteins Beclin and autophagy related 5 as well as the decreased the accumulation of $\mathrm{p} 62$, and increased the LC3II/I ratio. Sanggenon C also reduced hypoxia-induced apoptosis as detected by TUNEL staining and the expression of Bcl-2 proteins. The beneficial effects of Sanggenon $\mathrm{C}$ were associated with enhanced activation level of AMPK $\alpha$ and suppressed hypoxia-induced mechanistic target of rapamycin
\end{abstract}

Correspondence to: Professor Xiwen Zhang, Department of Cardiology, Huai'an First People's Hospital, Nanjing Medical University, 6 Beijing Road West, Huai'an, Jiangsu 223300, P.R. China E-mail: guyang1028@163.com

Key-words: Sanggenon C, cardiomyocyte, hypoxia, autophagy, AMP-activated protein kinase $\alpha$
(mTOR) and forkhead box O3a (FOXO3a) phosphorylation. The AMPK inhibitor Compound $\mathrm{C}(\mathrm{CpC})$ was used, and the anti-apoptotic and pro-autophagy effects of Sanggenon $\mathrm{C}$ in response to hypoxia were abolished by $\mathrm{CpC}$. In conclusion, the current study demonstrated that Sanggenon C possessed direct cytoprotective effects against hypoxia injury in cardiac cells via signaling mechanisms involving the activation of AMPK and concomitant inhibition of mTOR and FOXO3a.

\section{Introduction}

Acute myocardial infarction (AMI) results from a disruption of coronary blood flow to the myocardial region that it supplies. AMI remains the leading cause of mortality and is associated with a heavy financial burden worldwide (1). Prolonged cardiac ischemia can provoke tissue damage due to a lack of oxygen and nutrients since the heart demands high energy to function. The continuous deficiency of oxygen and nutrients alters ion homeostasis and metabolism, reducing cardiac contractility and structural organization, initiating cell death via necrosis and apoptosis (2). Cell death via necrosis is characterized by cell membrane rupture and the consequent release of cellular components (e.g., creatine kinase, troponin), which subsequently provokes an inflammatory response (3). The molecular mechanisms regarding ischemic injury are multifactorial. A growing number of studies have investigated the role that reactive oxygen species (ROS) serve in hypoxia, ranging from beneficial to damaging (4,5). At the basal level, ROS functions as a mediator for multiple cellular signaling cascades including cell growth and stress adaptation. In myocardial ischemia, excess ROS can damage tissues by oxidizing important cellular components such as proteins, lipids and DNA (4).

More recently, the role of autophagy in the pathogenesis of myocardial ischemic injury has been investigated. Autophagy is an evolutionarily conserved catabolic process that targets dysfunctional or damaged cytoplasmic constituents to the lysosome for degradation and recycling (6). Autophagy is essential for the survival of newborn mice between birth and the onset of suckling $(7,8)$. Previous research has demonstrated that HL-1 cells overexpressing green fluorescent protein (GFP)-microtubule-associated proteins 1A/1B light chain 3 (LC3) exposed to $2 \mathrm{~h}$ of simulated ischemia in the 
absence of oxygen exhibited a low level of autophagy (9) Hamacher-Brady et al (9) reported that autophagy was completely blocked in HL-1 cells exposed to $2 \mathrm{~h}$ of simulated ischemia in the absence of oxygen. Inhibition of autophagy was achieved by treatments with either 3-methyladenine or wortmannin, downregulation of Beclin 1 or overexpression of autophagy related 5 (Atg5) K130R; all these interventions sensitized HL-1 cardiac cells to apoptosis induced by ischemia reperfusion. These indicate the cardioprotective effect of autophagy against ischemia injury. Considerable advances have been made in recognizing the molecular mechanisms that determine the protection afforded by these strategies. Pharmacological agents targeting key signaling effectors involved in the ischemic and reperfusion cascades have also been developed (10), while most of them have yielded disappointing results in clinical trials (11). Despite these setbacks, identifying novel potential 'druggable' targets for the clinical management of AMI is remains imminent.

Sanggenon C, a flavanone Diel-Alder adduct compound, is isolated from the root bark of Morus cathayana. A previous study has reported that by inhibiting proteasome function, Sanggenon C inhibits tumor cell viability via induction of cell cycle arrest and cell death (12). Sanggenon C also protected against lipopolysaccharide (LPS)-stimulated RAW264.7-cell inflammation by inhibiting nitric oxide (NO) production and inducible nitric oxide synthase expression via suppressing nuclear factor $-\kappa \mathrm{B}(\mathrm{NF}-\kappa \mathrm{B})$ activity and $N F-\kappa \mathrm{B}$ inhibitor $\alpha$ activation (13). By suppressing the activation of $N F-\kappa B$, Sanggenon $C$ inhibited tumor necrosis factor $\alpha(\mathrm{TNF} \alpha)$ stimulated human polymorphonuclear leukocyte adhesion to human synovial cell and expression of vascular cell adhesion molecule 1 (14). Since Sanggenon C possesses antioxidant and anti-inflammatory activities, it may serve as a cardioprotective agent. The purpose of our study was to determine the effects of Sanggenon $\mathrm{C}$ on cardiomyocyte hypoxia injury.

\section{Materials and methods}

Reagents. The primary antibodies included phosphorylated AMP-activated protein kinase $\alpha$ (p-AMPK $\alpha$; cat. no. 2535), total (T)-AMPK $\alpha$ (cat. no. 2603P), p-mechanistic target of rapamycin (mTOR; cat. no. 2971), T-mTOR (cat. no. 2983), p-forkhead box O3a (FOXO3a; cat. no. 9465P), T-FOXO3a (cat. no. 2497P), Bcl-2 associated X apoptosis regulator (Bax; cat. no. 2722), Bcl-2 apoptosis regulator (Bcl-2; cat. no. 2870), and GAPDH (cat. no. 2118; all purchased from Cell Signaling Technology, Inc., Danvers, MA, USA). Sanggenon C (98\% purity as determined by high-performance liquid chromatography analysis) was purchased from Shanghai Winherb Medical S\&T Development Co., Ltd. (Shanghai, China). Fetal bovine serum was ordered from Gibco (Thermo Fisher Scientific, Inc., Waltham, MA, USA). The cell culture reagents were purchased from Gibco (Thermo Fisher Scientific, Inc.).

Cell culture. Rat cardiac H9c2 cells (Cell Bank of the Chinese Academy of Sciences, Shanghai, China) were cultured in Dulbecco's modified Eagle's medium (DMEM; cat. no. C11885; Gibco; Thermo Fisher Scientific, Inc.) supplemented with $10 \%$ fetal bovine serum (FBS; cat. no. 10099-133; Gibco; Thermo Fisher Scientific, Inc.,), $100 \mathrm{U} / \mathrm{ml}$ penicillin/100 mg/ml streptomycin (cat. no. 15140; Gibco; Thermo Fisher Scientific, Inc.) and $5 \% \mathrm{CO}_{2}$ at $37^{\circ} \mathrm{C}$. The media was changed every $1-2$ days and subcultured to $70-80 \%$ confluency. Cells were plated at an appropriate density according to each experimental design. Cells were seeded at a density of $1 \times 10^{6} /$ well onto 6 -well culture plates for mRNA extraction, $5 \times 10^{3} /$ well in 24 -well plates for cell surface area examination and $10 \times 10^{7} /$ well onto $10-\mathrm{cm}$ diameter culture plates for protein extraction. Following $24 \mathrm{~h}$ adherence, the culture medium was changed to serum-free DMEM $12 \mathrm{~h}$ prior to the experiment. Cells were pretreated with Sanggenon C (1, 10 and $100 \mu \mathrm{M})$ and/or Compound C $(\mathrm{CpC} ; 20 \mu \mathrm{M})$ for $12 \mathrm{~h}$ in serum-free DMEM at $37^{\circ} \mathrm{C}$ and then cells were maintained at $37^{\circ} \mathrm{C}$, under a hypoxic atmosphere of $95 \% \mathrm{~N}_{2}$ and $5 \% \mathrm{CO}_{2}$ for $24 \mathrm{~h}$.

Reverse transcription-quantitative polymerase chain reaction $(R T-q P C R)$. Total RNA was extracted from frozen H9c2 cells using TRIzol (cat.no. 15596-026; Thermo Fisher Scientific,Inc.). RNA yield and purity were evaluated using a SmartSpec Plus Spectrophotometer (Bio-Rad Laboratories Inc., Hercules, CA, USA), comparing the absorbance (A) 260/A280 and A230/260 ratios. RT was performed on RNA ( $2 \mu \mathrm{g}$ of each sample) to produce cDNA using oligo (dT) primers and the Transcriptor First Strand cDNA Synthesis kit (cat. no. 04896866001; Roche Diagnostics, Basel Switzerland). The PCR products were quantified using a LightCycler 480 SYBR-Green 1 Master mix (cat. no. 04707516001; Roche Diagnostics). Following an initial 5 min denaturation step at $95^{\circ} \mathrm{C}$, a total of 42 primer-extension cycles were carried out. Each cycle consisted of a $10 \mathrm{sec}$ denaturation step at $95^{\circ} \mathrm{C}$, a $20 \mathrm{sec}$ annealing step at $60^{\circ} \mathrm{C}$, and a $20 \mathrm{sec}$ incubation at $72^{\circ} \mathrm{C}$ for extension. Then a final extension step was performed at $72^{\circ} \mathrm{C}$ for $10 \mathrm{~min}$. The double standard curve was used to quantify the PCR results. Calibrator normalized ratio $=($ concentration of sample target/concentrations of sample reference)/(concentration of calibrator target/concentration of calibrator reference) (15). The results were normalized against GAPDH gene expression. The sequences of the oligonucleotide primers (Sangon Biotech Co., Ltd., Shanghai, China) were as follows: TNF $\alpha$ forward, 5'-AGCATGATCCGAGAT GTGGAA-3' and reverse, 5'-TAGACAGAAGAGCGTGGT GGC-3'; interleukin-1 (IL-1) forward, 5'-GGGATGATGACG ACCTGCTAG-3' and reverse, 5'-ACCACTTGTTGGCTTATG TTCTG-3'; IL-6 forward, 5'-GTTGCCTTCTTGGGACTG ATG-3' and reverse, 5'-ATACTGGTCTGTTGTGGGTGGT-3'; Beclin-1 forward, 5'-AGCTTTTCTGGACTGTGTGC-3' and reverse, 5'-TGAACTTGAGCGCCTTTGTC-3'; Atg5 forward, 5'-CAAGGATGCAGTTGAGGCTC-3' and reverse, 5'-AGT TTCCGGTTGATGGTCCA-3'; p62 forward, 5'-AAGAGG CTCCATCACCAGAG-3' and reverse, 5'-CCCCTTGACTCT GGCTGTAA-3'.

ROS measurement. The level of intracellular ROS generation was assessed using the fluorescent dye 2',7'-dichlorofluorescin diacetate (DCFH-DA). Following the indicated treatments, cells were washed twice with PBS and then incubated with serum-free DMEM and $1 \times 10^{-5} \mathrm{~mol} / 1 \mathrm{DCFH}-\mathrm{DA}$ in a $37^{\circ} \mathrm{C}$ incubator for $30 \mathrm{~min}$. Subsequently, cells were washed with PBS for three times to eliminate the residual DCFH-DA. A fluorescence microscope (BX51; Olympus Corporation, Tokyo, Japan) was also used to evaluate the DCFH florescence of cells on coverslips. 
Antioxidative assessments. Antioxidative capacity was assessed by the release of NO and the activity of SOD according to the protocol of the NO detection kit, SOD detection kit, respectively (Beyotime Institute of Biotechnology, Haimen, China). For NO detection, the supernatant was collected and transferred to another 96-well plate. The NO released from $\mathrm{H} 9 \mathrm{c} 2$ cells was measured at $540 \mathrm{~nm}$ using spectrophotometer according to the manufacturer's instructions (Beyotime Institute of Biotechnology). For SOD assessment, proteins were extracted and quantified before measuring the activity of SOD at $450 \mathrm{~nm}$ using the commercial kit (Beyotime Institute of Biotechnology).

Terminal deoxynucleotidyl-transferase-mediated dUTP nick-end labelling (TUNEL) staining. Cells were cultured on cover slips in a 24 -well plate, fixed in $4 \%$ paraformaldehyde for $5 \mathrm{~min}$ at room temperature and then permeabilized in $0.1 \%$ Triton $\mathrm{X}-100$ for $5 \mathrm{~min}$ in room temperature following treatment. TUNEL staining according to the protocol of ApopTag ${ }^{\circledR}$ Plus Fluorescein in situ Apoptosis Detection kit (EMD Millipore, Billerica, MA, USA) for $1 \mathrm{~h}$ at $37^{\circ} \mathrm{C}$. Nuclei were labeled with DAPI and DNA fragmentation was quantified under fluorescence microscope (magnification, x200; BX51TRF; Olympus Corporation). The percentages of TUNEL-positive cells relative to DAPI-positive cells were calculated by an investigator in a blinded manner.

Western blot analysis. Cultured cardiac H9c2 cells were lysed in radioimmunoprecipitation (RIPA) lysis buffer [720 $\mu$ l RIPA, $20 \mu$ l phenylmethylsulfonyl fluoride $(1 \mathrm{mM}), 100 \mu \mathrm{l}$ cOmplete $^{\mathrm{TM}}$ protease inhibitor cocktail (cat. no. 04693124001; Sigma-Aldrich; Merck KGaA, Darmstadt, Germany)], $100 \mu \mathrm{l}$ phosSTOP (cat. no. 04906837001; Roche Diagnostics), $50 \mu \mathrm{l}$ $\mathrm{NaF}(1 \mathrm{mM}), 10 \mu 1 \mathrm{Na}_{3} \mathrm{VO}_{4} / \mathrm{ml}$ and the protein concentration was measured by the bincinchoninic assay method. A total of $30 \mu \mathrm{g}$ cell lysate was used for protein separation using SDS-PAGE on a $10 \%$ gel. The proteins were then transferred to polyvinylidene difluoride (PVDF) membranes (EMD Millipore). Specific protein expression levels were normalized to the GAPDH protein levels of the total cell lysate and cytosolic proteins on the same PVDF membranes, which were blocked with 5\% non-fat milk at room temperature for $2 \mathrm{~h}$. The following primary antibodies were used: p-AMPK $\alpha$, T-AMPK $\alpha$, p-mTOR, T-mTOR, p-FOXO3a, T-FOXO3a, Bax, Bcl-2, and GAPDH. The primary antibodies were diluted at 1:1,000. Antibody incubation was performed overnight with gentle shaking at $4^{\circ} \mathrm{C}$. Quantification of the western blots was performed using an Odyssey infrared imaging system (LI-COR, Lincoln, NE, USA). The secondary antibodies, goat anti-rabbit IRdye ${ }^{\circledR} 800 \mathrm{CW}$ (cat. no. 926-32211; LI-COR) IgG and goat anti-mouse IRdye $800 \mathrm{CW}$ (cat. no. 926-32210; LI-COR), were used at a 1:10,000 dilution at $37^{\circ} \mathrm{C}$ in Odyssey blocking for $1 \mathrm{~h}$. The blots were scanned using an infrared LI-COR scanner, allowing for simultaneous detection of two targets (phosphorylated and total protein) within the same experiment.

Statistical analysis. Data is expressed as the mean \pm standard error of the mean. Differences among groups were determined by a two-way analysis of variance followed by Tukey's post
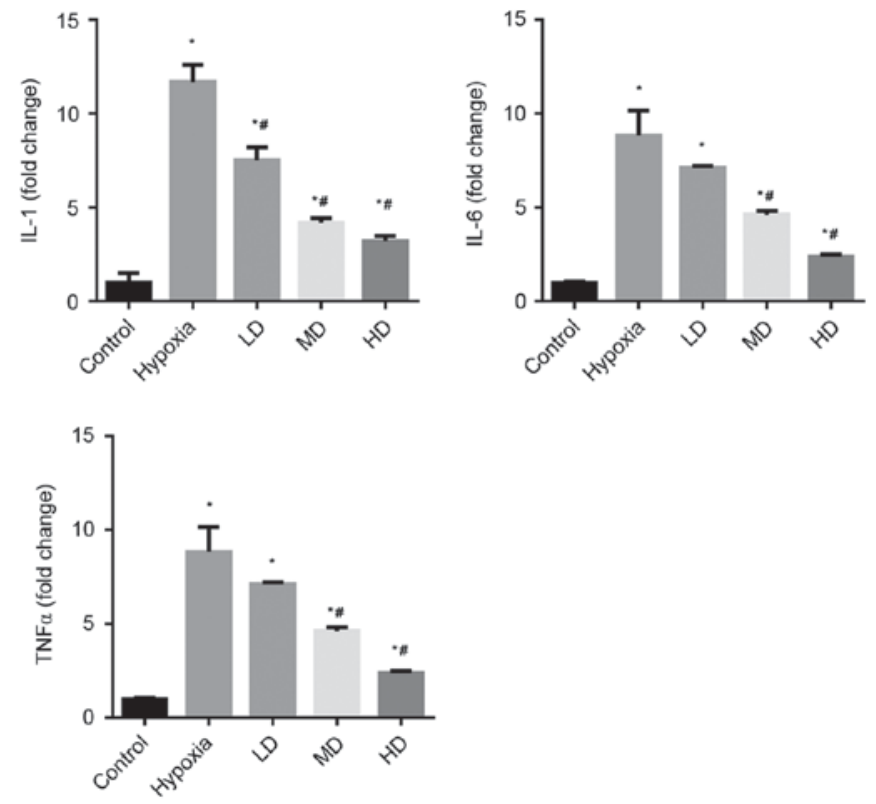

Figure 1. Sanggenon C suppresses hypoxia-induced inflammation in cardiomyocytes. Reverse transcription-quantitative polymerase chain analysis of the mRNA levels of IL-1, IL-6, and TNF $\alpha$ induced by Sanggenon C $(1,10$ and $100 \mu \mathrm{M})$ after hypoxia for $24 \mathrm{~h}(\mathrm{n}=6) .{ }^{*} \mathrm{P}<0.05$ vs. control group; ${ }^{*} \mathrm{P}<0.05$ vs. hypoxia group. LD, low dose $1 \mu \mathrm{M}$ Sanggenon C; MD, mid dose $10 \mu \mathrm{M}$ Sanggenon C; HD, high dose $100 \mu \mathrm{M}$ Sanggenon C; IL, interleukin; TNFo, tumor necrosis factor $\alpha$.

hoc test. Statistical analyses were conducted using SPSS software (version 19.0; IBM Corp., Armonk, NY, USA). P<0.05 was considered to indicate a statistically significant difference.

\section{Results}

Sanggenon $C$ suppresses hypoxia-induced inflammation in cardiomyocytes. The effects of Sanggenon $C$ on the induction of TNF $\alpha$, IL- $1 \beta$ and IL- 6 in cardiomyocytes exposed to hypoxia were measured by RT-qPCR. The expression levels of pro-inflammatory cytokines TNF $\alpha$, IL- $1 \beta$ and IL- 6 were significantly increased in the hypoxia group. Sanggenon C treatment significantly attenuated this increase in a concentration-dependent manner (Fig. 1).

Sanggenon C inhibits the oxidative stress induced by hypoxia in cardiomyocytes. Cells incubated with DCFH-DA were used to determine the ROS production. A marked increase of ROS was observed in H9c2 cells exposed to hypoxia, while $100 \mu \mathrm{M}$ Sanggenon C suppressed hypoxia-induced ROS generation. The effect of Sanggenon C on the release of antioxidants was also measured. Exposure to hypoxia for $24 \mathrm{~h}$ significantly decreased the release of NO and SOD activity in H9c2 cells and $100 \mu \mathrm{M}$ Sanggenon $\mathrm{C}$ significantly attenuated this decrease (Fig. 2A and B).

Sanggenon C activates autophagy in response to hypoxia in cardiomyocytes. The effect of Sanggenon C on cardiomyocyte autophagy in response to hypoxia was explored. Previous studies have demonstrated that Beclin-1, p62, Atg5 and LC3 are autophagy-associated proteins and enhanced autophagy is accompanied by an increased ratio of LC3 II/LC3 I and 


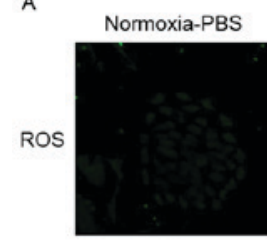

B

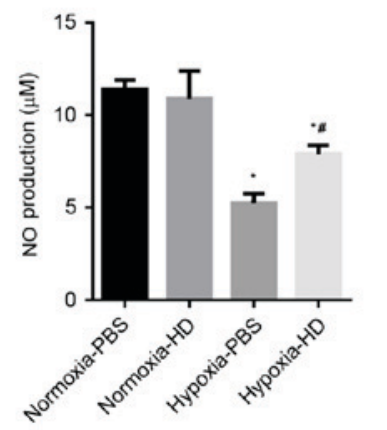

Hypoxia-PBS

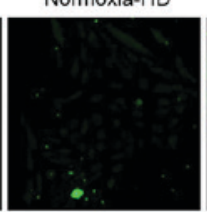

Hypoxia-HD
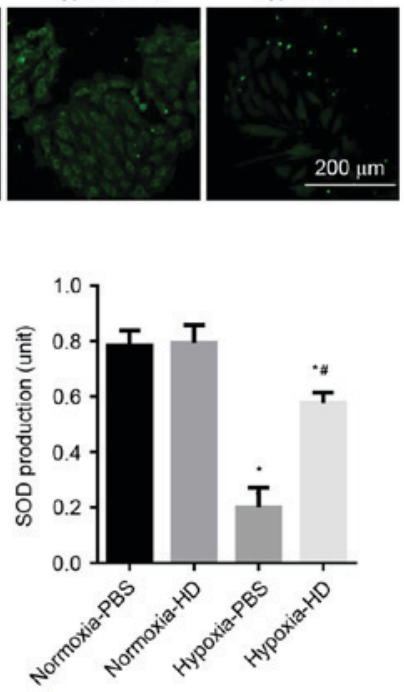

Figure 2. Sanggenon $\mathrm{C}$ inhibits the oxidative stress induced by hypoxia in cardiomyocytes. (A) Sanggenon C (100 $\mu \mathrm{M})$ inhibits the ROS production induced by hypoxia which was detected by florescence microscope, $(\mathrm{n}=3)$. (B) Effect of Sanggenon C $(100 \mu \mathrm{M})$ on the production of NO and activity of SOD of hypoxia cardiomyocyte were determined by kit $(n=6)$. ${ }^{*} \mathrm{P}<0.05$ vs. normoxia-PBS; ${ }^{\#} \mathrm{P}<0.05$ vs. hypoxia-PBS. HD, high dose $100 \mu \mathrm{M}$ Sanggenon C; ROS, reactive oxygen species; SOD, super oxide dismutase.
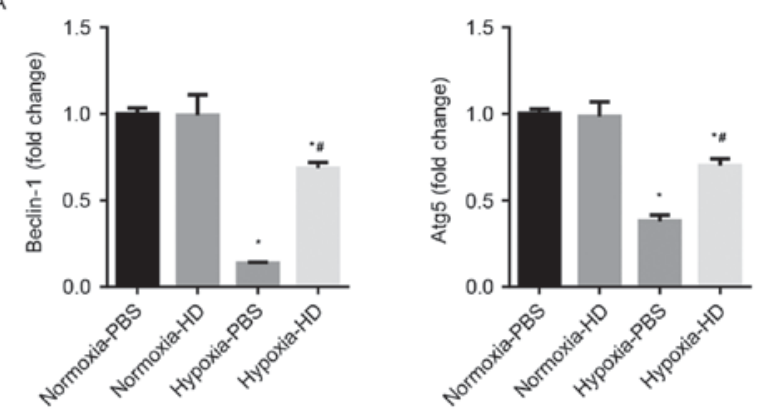

B
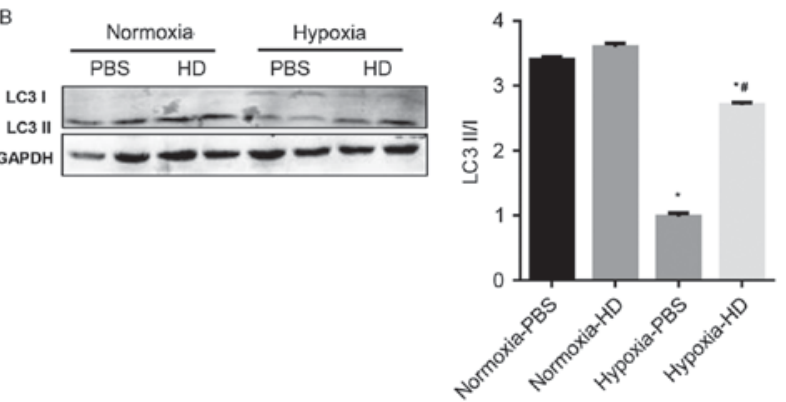

Figure 3. Sanggenon $\mathrm{C}$ activates autophagy in response to hypoxia in cardiomyocytes (A) Reverse transcription-quantitative polymerase chain reaction analysis of the mRNA levels of Atg5 and Beclin-1 in the indicated group $(n=6)$. (B) Western blot analysis the effect of Sanggenon C $(100 \mu \mathrm{M})$ on the transformation of LC3 I to LC3 II ( $n=6)$. Left, representative blots. Right, quantitative results. ${ }^{*} \mathrm{P}<0.05$ vs. normoxia-PBS; ${ }^{\#} \mathrm{P}<0.05$ vs. hypoxia-PBS. $\mathrm{HD}$, high dose $100 \mu \mathrm{M}$ Sanggenon C; Atg5, autophagy related 5; LC3, microtubule-associated proteins $1 \mathrm{~A} / 1 \mathrm{~B}$ light chain 3 .

Atg5 and Beclin-1 expression (16). The results demonstrated that autophagy significantly decreased in hypoxia-exposed cardiomyocytes, however increased in $100 \mu \mathrm{M}$ Sanggenon $\mathrm{C}$ pre-treated cardiomyocytes (Fig. 3A and B).

Sanggenon $C$ attenuates hypoxia-induced apoptosis in cardiomyocytes. To demonstrate the effect of Sanggenon $\mathrm{C}$ on cardiomyocyte apoptosis in response to hypoxia, TUNEL staining and western blotting were performed to detect cell apoptosis. Results revealed that the rate of apoptotic cells increased significantly after 24 h of hypoxia. Sanggenon C significantly reduced hypoxia-induced apoptosis (Fig. 4A and B). As expected, changes in expression of apoptosis-associated proteins were also consistent with the results above (Fig. 4C and D).

Effect of Sanggenon C on AMPKa/mTOR/FOXO3a signaling. Cellular energy levels are associated with the autophagy machinery by means of three major energy sensing pathways: AMPK, mTOR, and FOXO3a (16). In the current study, activated AMPK $\alpha$ was decreased, and phosphorylated mTOR and FOXO3a were increased in cardiomyocytes under hypoxia. Sanggenon $\mathrm{C}$ pre-treatment significantly increased the activation of AMPK $\alpha$, and reduced the phosphorylation of mTOR and FOXO3a (Fig. 5A and B). To confirm the protective effects of Sanggenon $C$ on hypoxia-induced cardiomyocyte injury were mediated by AMPK $\alpha$ signaling, an AMPK $\alpha$ inhibitor, $\mathrm{CpC}(20 \mu \mathrm{M})$ was used. The anti-apoptotic and pro-autophagy effects of Sanggenon $\mathrm{C}$ in response to hypoxia were abolished by $\mathrm{CpC}$ (Fig. 5C-E). The data indicated that enhanced autophagy and reduced inflammation, oxidative stress, apoptosis in Sanggenon C-pretreated cardiomyocytes exposed to hypoxia may be mediated through the regulation of the AMPK $\alpha /$ mTOR/FOXO3a signaling pathway.

\section{Discussion}

In the present study, it was demonstrated that Sanggenon C could increase cardiomyocyte autophagy in hypoxic conditions and prevent myocyte apoptosis. The increase in autophagy was associated with ROS clearance and the inhibition of inflammation, which would lead to cell death. The cardiac-protective effect of Sanggenon $\mathrm{C}$ was mediated by the modulation of AMPK $\alpha / m$ TOR/FOXO3a signaling.

In myocardial infarction and cardiomyocyte hypoxia, necrotic cardiomyocytes are capable of releasing a wide range of damage-associated molecular signals that activate innate immune pathways triggering an inflammatory response (17). Although inflammation is required for the phagocytotic removal of dead cells and for the activation of reparative mesenchymal cells, overactive, dysregulated, temporally-prolonged, or spatially expanded inflammatory responses may cause death of viable cardiomyocytes. This may also enhance matrix degradation (thus promoting dilative remodeling) and extend fibrosis (18). The oxidant and antioxidant imbalance within cardiomyocytes, which favors the accumulation of oxidants, can lead to cellular damage that constitutes oxidative stress (19). In the healthy myocardium, ROS is an unintended byproduct of mitochondrial respiration, where its concentration is tightly controlled to a low steady state level by antioxidants. However, in the ischemic heart, NO', $\mathrm{O}_{2}{ }^{-}$, and $\mathrm{NO}_{3}{ }^{-}$formation are elevated. The electron leakage from complexes I and III of the electron transport chain is primarily responsible for $\mathrm{O}_{2}{ }^{-}$generation in mitochondria. This in turn damages the inner mitochondrial membrane, leading 


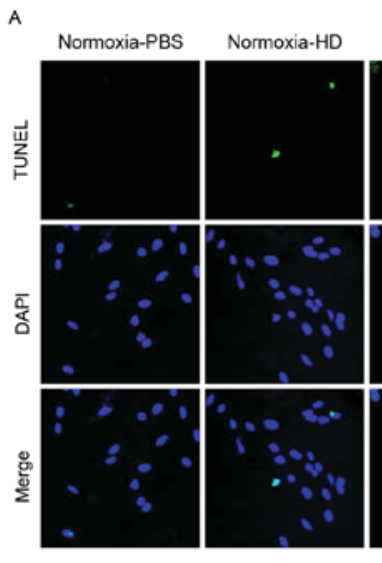

C

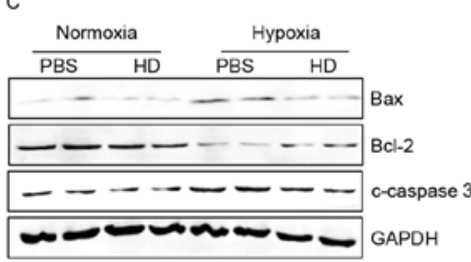

Hypoxia-PBS

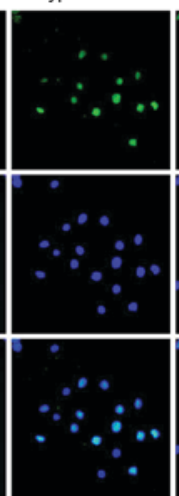

Hypoxia-HD

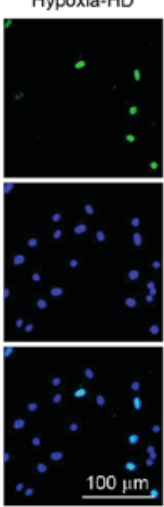

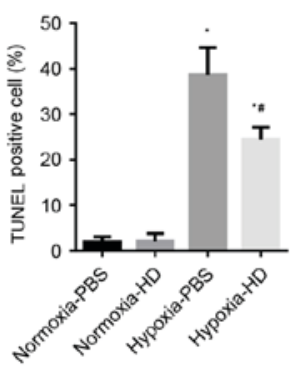

Figure 4. Sanggenon C attenuates hypoxia-induced apoptosis in cardiomyocytes. (A) Effect of Sanggenon C (100 $\mu \mathrm{M})$ on the hypoxia-induced apoptosis among cardiomyocytes detected by TUNEL staining and (B) quantification (n=3). (C) The effect of Sanggenon $\mathrm{C}(100 \mu \mathrm{M})$ on the activation of apoptotic signaling pathways including Bax and Bcl-2 determined by western blot analysis and (D) densitometry semi-quantification ( $\mathrm{n}=6$ ). ${ }^{*} \mathrm{P}<0.05$ vs. normoxia-PBS; ${ }^{\#} \mathrm{P}<0.05$ vs. hypoxia-PBS. HD, high dose $100 \mu \mathrm{M}$ Sanggenon C; TUNEL, terminal deoxynucleotidyl-transferase-mediated dUTP nick-end labelling; Bax, Bcl-2 associated $\mathrm{X}$ apoptosis regulator; $\mathrm{Bcl}-2$, Bcl-2 apoptosis regulator; c-caspase 3, cleaved caspase 3.
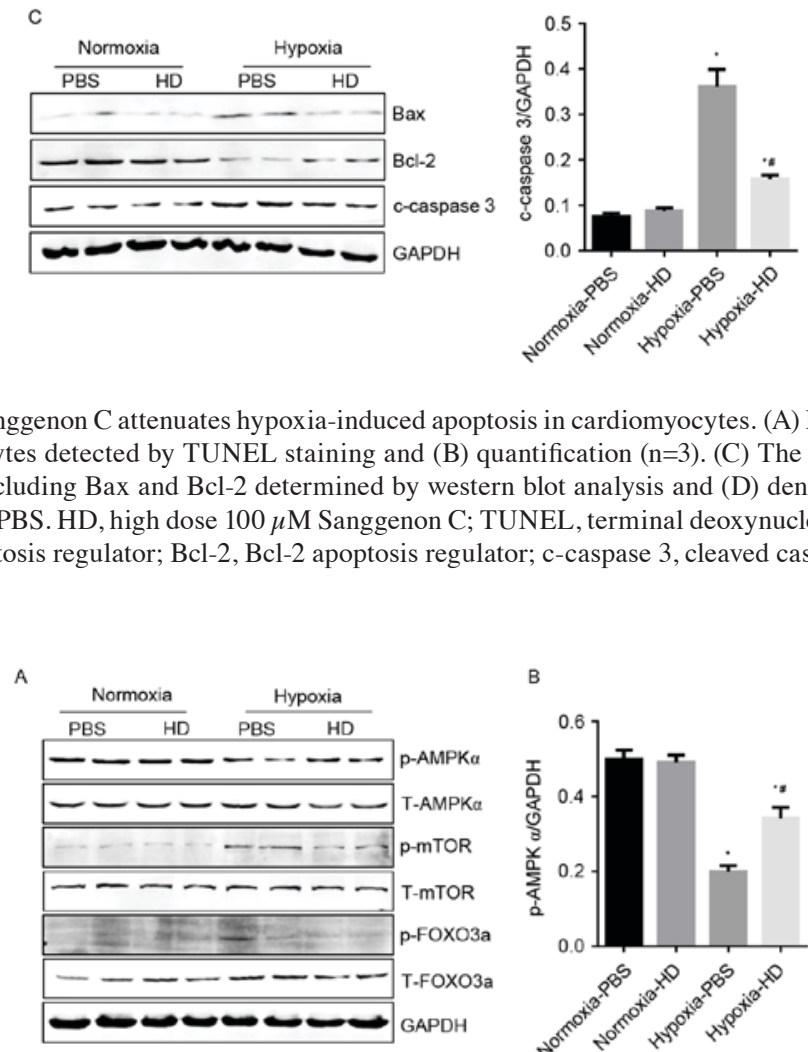

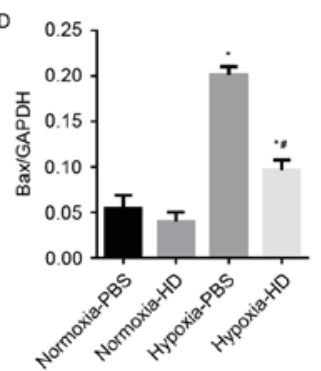

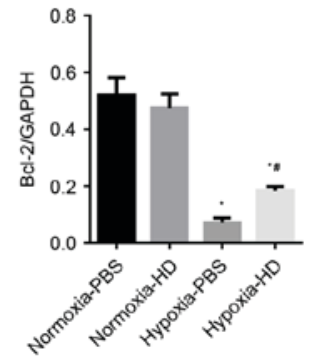
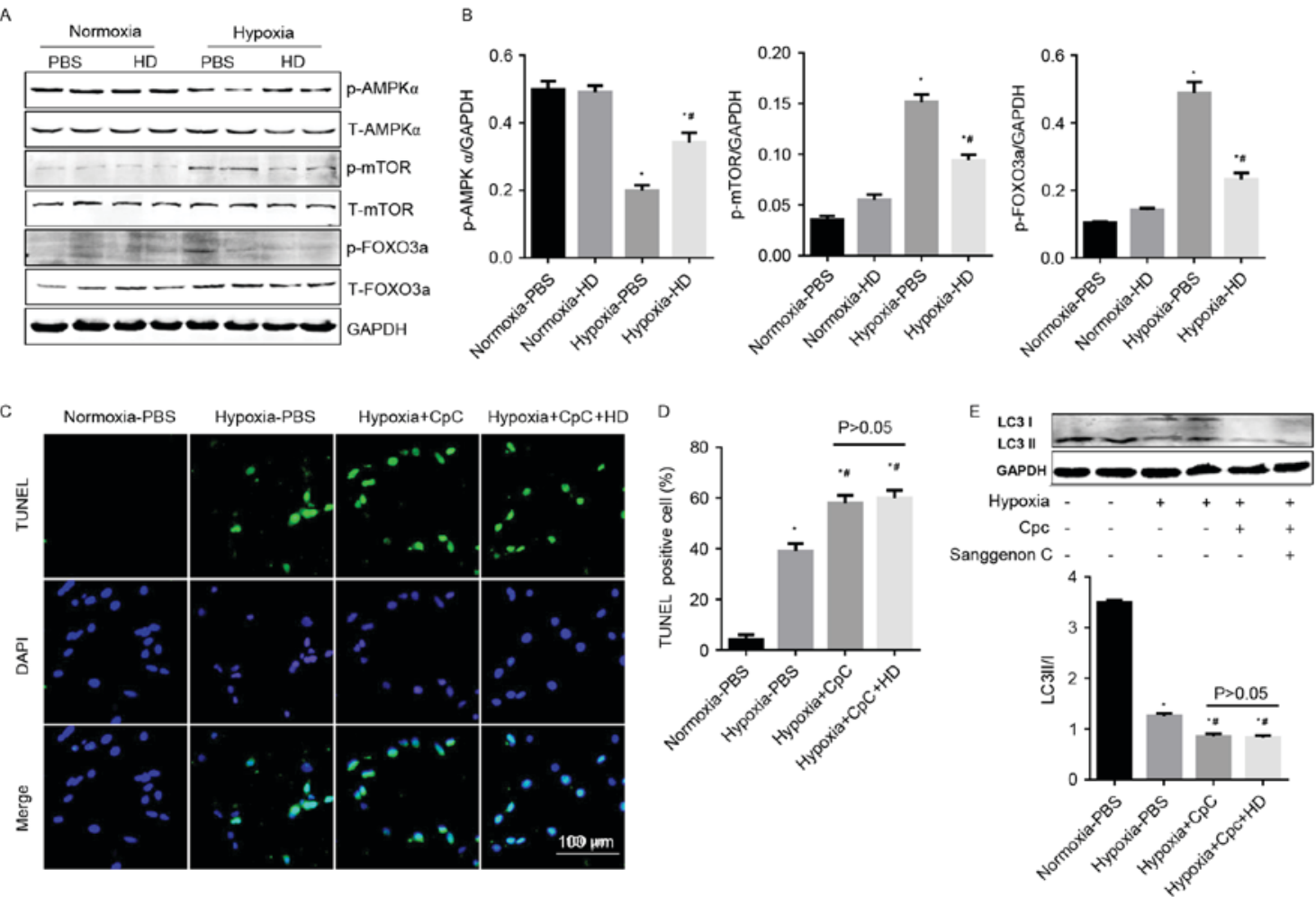

Figure 5. Effect of Sanggenon C on AMPKa/mTOR/FOXO3a signaling. (A) Western blot analysis the effect of Sanggenon C (100 $\mu \mathrm{M})$ on the activation of AMPK $\alpha$ pathways including phosphorylated (p-) and total (T-) AMPK $\alpha$, mTOR and FOXO3a with (B) densitometry analysis (n=6). (C) TUNEL staining of cardiomyocytes pretreated with Sanggenon C $(100 \mu \mathrm{M})$ or AMPKa inhibitor, CpC $(20 \mu \mathrm{M})$ and exposed to hypoxia for $24 \mathrm{~h}(\mathrm{n}=3)$ and $(\mathrm{D})$ quantification of TUNEL-positive cells. (E) Western blot analysis the effect of Sanggenon C $(100 \mu \mathrm{M})$ and CpC on the transformation of LC3 I to LC3 II (n=6). "P<0.05 vs. normoxia-PBS; "P $<0.05$ vs. hypoxia-PBS. HD, high dose $100 \mu \mathrm{M}$ Sanggenon C; p-, phospho; T-, total; AMPK $\alpha$, AMP-activated protein kinase $\alpha$; mTOR, mechanistic target of rapamycin; FOXO3a, forkhead box O3a; TUNEL, terminal deoxynucleotidyl-transferase-mediated dUTP nick-end labeling; LC3, microtubule-associated proteins $1 \mathrm{~A} / 1 \mathrm{~B}$ light chain 3 ; $\mathrm{CpC}$, compound $\mathrm{C}$. 
to decreased ATP production and then subsequently cellular damage (5). Previous studies have reported that Sanggenon C possesses antioxidant and anti-inflammatory activities $(12,13)$. In addition to this, the present study demonstrated that Sanggenon C reduced the expression of pro-inflammatory cytokines, decreased ROS generation and increased the antioxidant production in cardiomyocytes in response to hypoxia.

Autophagy involves many actions that are essential for cell survival. It preserves energy availability and removes damaged organelles through limited cellular catabolism. Removal of damaged mitochondria is particularly important since these organelles produce ROS and contribute to cell stress and damage. Autophagy reduces pro-inflammatory signals by eliminating intracellular organisms, degrading pro-inflammatory signaling platforms, and by controlling cytokine production and release. Studies have demonstrated that cells exposed to $2 \mathrm{~h}$ of simulated ischemia in the absence of oxygen exhibited a low level of autophagy $(9,20)$. It is widely agreed that autophagy elicited by myocardial infarction protects the heart from ischemic injury. Due to the abrupt interruption of exogenous nutrient supply, metabolites from autophagic digestion become a major source for energy production (21). Stimulation of autophagy in mice using TAT-p27 fusion protein reduces infarct size and improves cardiac performance following myocardial infarction (22). Furthermore, elevated basal levels of autophagy in communities that live at high altitudes are associated with diminished ischemia-reperfusion injury (23). The results of the current showed that autophagy reduced sharply after $24 \mathrm{~h}$ of hypoxia, whereas Sanggenon C-pretreatment induced cardiomyocyte autophagy. Increased autophagy in the Sanggenon C-treated cardiomyocyte may contribute to the protection against hypoxia.

To investigate the mechanism of induced autophagy following Sanggenon C treatment, the signal pathway associated with autophagy was detected. Autophagy is regulated by multiple signaling pathways, involving nutrients, stress, hormones, growth factors and intracellular energy information (8). AMPK, a sensor of the intracellular AMP/ATP ratio, is activated in response to elevated intracellular AMP by ATP hydrolysis. In conditions when ATP is depleted, AMPK is activated and subsequently phosphorylates eukaryotic elongation factor-2 kinase. This leads to a balance between the induction of autophagy and the inhibition of peptide elongation (24). AMPK also suppresses mTOR activity by interfering with GTPase activity, leading to the activation of autophagy (8). mTOR, a sensor of nutrients, is repressed under conditions of nutrient deprivation and hypoxia. Repression of mTOR promotes increased autophagic activity (25). FOXO3a, an evolutionarily conserved subfamily of transcription factors involved in regulation of energy metabolism, is also modulated by AMPK. Activation of the FOXO3a transcriptional program initially induces autophagy as an attempt to retain energy for cell survival (26). Treatment with Sanggenon C significantly increased the activation of AMPK $\alpha$ and FOXO3a, but suppressed the activation of mTOR in hypoxia cardiomyocytes. These results suggested that the regulation of AMPK $\alpha / m T O R / F O X O 3 a$ signal pathway by Sanggenon C is the compensatory mechanisms that induce autophagy and attenuate hypoxia injury in cardiomyocytes.
In conclusion, the current results supported the theory that Sanggenon $\mathrm{C}$, administered as a pre-treatment prior to cardiomyocyte hypoxia, attenuates the inflammatory response and ROS production provoked during hypoxia. Notably, Sanggenon C was demonstrated to promote autophagy and render the cardiomyocyte resistant to hypoxic injury. The modulation of AMPK $\alpha / m$ TOR/FOXO3a signaling pathway restored autophagy as a reflex reaction.

\section{Acknowledgements}

This study was supported by the Applied and Technologic Research Program of Huai'an (grant no. HAS2014009) and the Research Fund for the Technology Development Project of Nanjing Medical University (grant no. 2013NJMU226).

\section{References}

1. Reed GW, Rossi JE and Cannon CP: Acute myocardial infarction. Lancet 389: 197-210, 2017.

2. Heusch G and Gersh BJ: The pathophysiology of acute myocardial infarction and strategies of protection beyond reperfusion: A continual challenge. Eur Heart J 38: 774-784, 2017.

3. Westman PC, Lipinski MJ, Luger D, Waksman R, Bonow RO, Wu E and Epstein SE: Inflammation as a driver of adverse left ventricular remodeling after acute myocardial infarction. J Am Coll Cardiol 67: 2050-2060, 2016.

4. Zhou T, Chuang CC and Zuo L: Molecular characterization of reactive oxygen species in myocardial ischemia-reperfusion injury. Biomed Res Int 2015: 864946, 2015.

5. Kurian GA, Rajagopal R, Vedantham S and Rajesh M: The role of oxidative stress in myocardial ischemia and reperfusion injury and remodeling: Revisited. Oxid Med Cell Longev 2016: $1656450,2016$.

6. Lippai M and Szatmári Z: Autophagy-from molecular mechanisms to clinical relevance. Cell Biol Toxicol 33: 145-168, 2016.

7. Nishida K and Otsu K: Autophagy during cardiac remodeling. J Mol Cell Cardiol 95: 11-18, 2016.

8. Chen-Scarabelli C, Agrawal PR, Saravolatz L, Abuniat C, Scarabelli G, Stephanou A, Loomba L, Narula J, Scarabelli TM and Knight $\mathrm{R}$ : The role and modulation of autophagy in experimental models of myocardial ischemia-reperfusion injury. J Geriatr Cardiol 11: 338-348, 2014.

9. Hamacher-Brady A, Brady NR, Logue SE, Sayen MR, Jinno M, Kirshenbaum LA, Gottlieb RA and Gustafsson AB: Response to myocardial ischemia/reperfusion injury involves Bnip3 and autophagy. Cell Death Differ 14: 146-157, 2007.

10. Kharbanda RK: Cardiac conditioning: A review of evolving strategies to reduce ischaemia-reperfusion injury. Heart 96: 1179-1186, 2010.

11. Downey JM and Cohen MV: Why do we still not have cardioprotective drugs? Circ J 73: 1171-1177, 2009.

12. Huang H, Liu N, Zhao K, Zhu C, Lu X, Li S, Lian W, Zhou P, Dong X, Zhao C, et al: Sanggenon C decreases tumor cell viability associated with proteasome inhibition. Front Biosci (Elite Ed) 3: 1315-1325, 2011.

13. Dat NT, Binh PT, Quynh le TP, Huong HT and Minh CV: Sanggenon $\mathrm{C}$ and $\mathrm{O}$ inhibit NO production, iNOS expression and NF- $\kappa$ B activation in LPS-induced RAW264.7 cells. Immunopharmacol Immunotoxicol 34: 84-88, 2012.

14. Li LC, Shen F, Hou Q and Cheng GF: Inhibitory effect and mechanism of action of Sanggenon $\mathrm{C}$ on human polymorphonuclear leukocyte adhesion to human synovial cells. Acta Pharmacol Sin 23: 138-142, 2002.

15. Livak KJ and Schmittgen TD: Analysis of relative gene expression data using real-time quantitative PCR and the 2(-Delta Delta C(T)) method. Methods 25: 402-408, 2001.

16. Nishida K, Kyoi S, Yamaguchi O, Sadoshima J and Otsu K: The role of autophagy in the heart. Cell Death Differ 16: 31-38, 2009.

17. Rienks $M$ and Papageorgiou AP: Novel regulators of cardiac inflammation: Matricellular proteins expand their repertoire. J Mol Cell Cardiol 91: 172-178, 2016.

18. Frangogiannis NG: Inflammation in cardiac injury, repair and regeneration. Curr Opin Cardiol 30: 240-245, 2015. 
19. Sun Y: Oxidative stress and cardiac repair/remodeling following infarction. Am J Med Sci 334: 197-205, 2007.

20. Hamacher-Brady A, Brady NR and Gottlieb RA: Enhancing macroautophagy protects against ischemia/reperfusion injury in cardiac myocytes. J Biol Chem 281: 29776-29787, 2006.

21. Wang ZV and Hill JA: Protein quality control and metabolism: Bidirectional control in the heart. Cell Metab 21: 215-226, 2015.

22. Sun X, Momen A, Wu J, Noyan H, Li R, von Harsdorf R and Husain M: p27 protein protects metabolically stressed cardiomyocytes from apoptosis by promoting autophagy. J Biol Chem 289: 16924-16935, 2014
23. Hu Y, Sun Q, Li Z, Chen J, Shen C, Song Y and Zhong Q: High basal level of autophagy in high-altitude residents attenuates myocardial ischemia-reperfusion injury. J Thorac Cardiovase Surg 148: 1674-1680, 2014.

24. Bairwa SC, Parajuli N and Dyck JR: The role of AMPK in cardiomyocyte health and survival. Biochim Biophys Acta 1862: 2199-2210, 2016.

25. Gallagher LE, Williamson LE and Chan EY: Advances in autophagy regulatory mechanisms. Cells 5: pii: E24, 2016.

26. Chiacchiera F and Simone C: The AMPK-FoxO3A axis as a target for cancer treatment. Cell Cycle 9: 1091-1096, 2010. 